\title{
Experimental Study on Film Cooling Effectiveness of Shallow Hole with Upstream Sister Holes
}

\author{
Hamidon Bin Salleh ${ }^{1, a}$ and Yee Jingzu ${ }^{1, b}$ \\ 11Department of Plant and Automotive Engineering, \\ Faculty of Mechanical and Manufacturing Engineering, \\ Universiti Tun Husseion Onn Malaysia, \\ 86400 Parit Raja, Batu Pahat, Johor Darul Takzim, Malaysia.

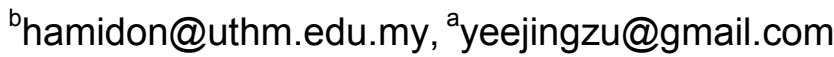

Keywords: Gas turbine cooling technology, film cooling, shallow hole, sister holes.

\begin{abstract}
In order to increase the thermal efficiency of a gas turbine, the operating temperature has to be increased. This increment may cause the material of the blade to melt. Film cooling is a good option to solve this problem. Various studies has been done on film cooling include the shallow hole and sister holes. The present study focused on an experimental of film cooling effectiveness on shallow hole of $20^{\circ}$ with upstream sister holes with 3 blowing ratios which are 1.0, 15 and 2.0. The result showed significant improvement compared to shallow hole of $35^{\circ}$. The optimum blowing ratio is 1.5. Smaller shallow angle and upstream sister holes reduce the jet lifting effect of the secondary air flow. Future study can be done on shallow hole of shallow angle and blowing ratio around 1.5 in order to further improve the film cooling effectiveness.
\end{abstract}

\section{Introduction}

Gas turbine, an internal combustion engine using air as working fluid, is widely used today in aircraft applications, power generation applications and other applications. According to Han et al. [1], in order to achieve a better performance in future, it is necessary to increase its thermal efficiency by increasing the turbine inlet temperature and also the compression ratio. Therefore, development of high-temperature material and highly sophisticated cooling scheme are essential. In study, the focus will be on the external turbine cooling, in other words, film cooling.

Goldstein [2] stated that film cooling is the introduction of a secondary fluid at one or more discrete locations along a surface exposed to a high temperature environment to protect that surface not only in the immediate region of injection but also in the downstream region. It is affected by few factors include pressure ratio and temperature ratio of coolant to mainstream, the location, configuration and distribution on the airfoil of the film cooling hole and the design of the film cooling holes.

Abdullah et al. [3] have done thermal and aerodynamics investigations of multiple cooling holes with shallow hole angle. A baseline test model, TMB is set up with $35^{\circ}$ hole angle cooling holes. Another two models, TMA and TMG, have shallow hole angle of $20^{\circ}$ with different lateral pitch distance of $6 \mathrm{D}$ and 3D respectively. For the test plate, 20 cooling holes arranged in $5 \times 4$ matrix. The experimental condition is Reynolds number of 6200 with blowing ratios of 0.5, 1.0 and 2.0. In-line hole arrangement superposition effect is detected from the studies which has increased the film cooling effectiveness of all test models at all blowing ratios. Only the film cooling effectiveness of TMB at blowing ratio of 0.5 is not increased. In other cases, higher blowing ratio usually reduces the film cooling performance. However, in this case, specifically for TMG, the film holes produce kidney vortices. The interactions provides a better film cooling coverage at $x / D>20$.

On the other hand, the study of Ely et al. [4] focused on a computational analysis on the effect of the sister hole control on film cooling from short holes. Short hole is a design of film cooling hole with the length to diameter ratio $(L / D)$ equal or less than one. It is liable to create complicated vortex structures. One significant difference between short hole and long hole is the flow fields 
created. The study is to determine the best position of sister holes to maintain flow adhesion along the surface of the blade actively. From the study, with sister holes, the short hole produces similar form as long hole. The near hole film cooling effectiveness can be improved by using upstream sister holes while the downstream film cooling effectiveness can be improved by using downstream sister holes.

As for the current study, it aims to investigate the film cooling effectiveness of the film holes with shallow angle of $20^{\circ}$ with upstream sister holes attached to the primary hole by using experimental method. with blowing ratios of $M=1.0,1.5$ and 2.0.

\section{Experimental Method}

Experimental Setup. The study is carried out by using experimental method. Figure 1 shows the schematic diagram of the experimental setup. The apparatus used are open loop low speed wind tunnel for mainstream air supply, Wind Power Air Blower for secondary flow air supply, air heater to heat up air from secondary flow, Fluke 922 is used with Pitot Tube and also Venturi Meter for flow metering, thermocouple, and thermometer and infrared camera are used for temperature measurement. A test rig is fabricated for the test section.

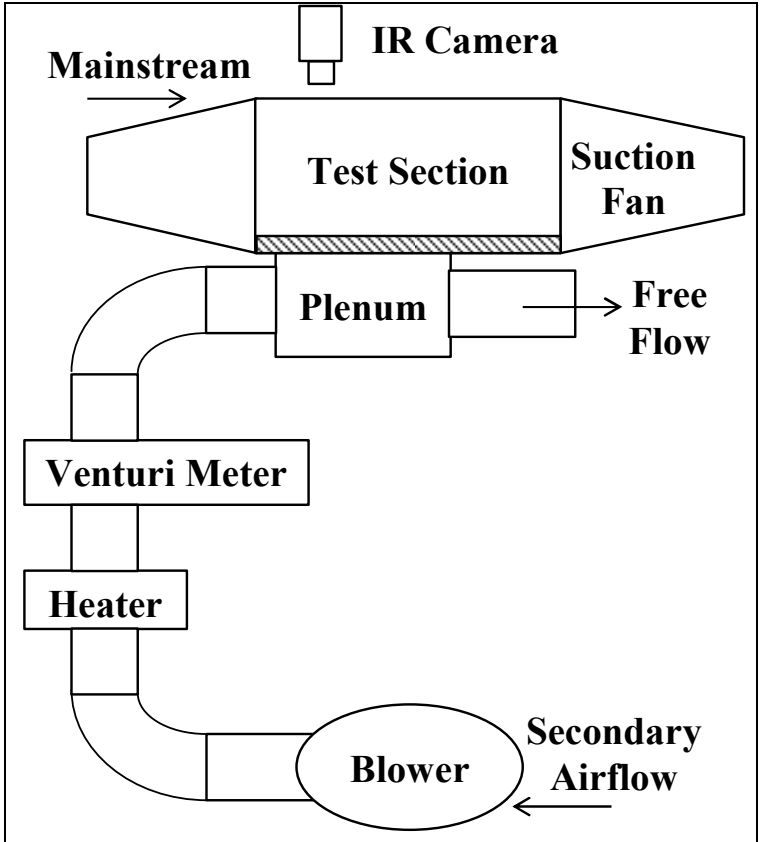

Fig. 1 Sketch of Experimental Setup

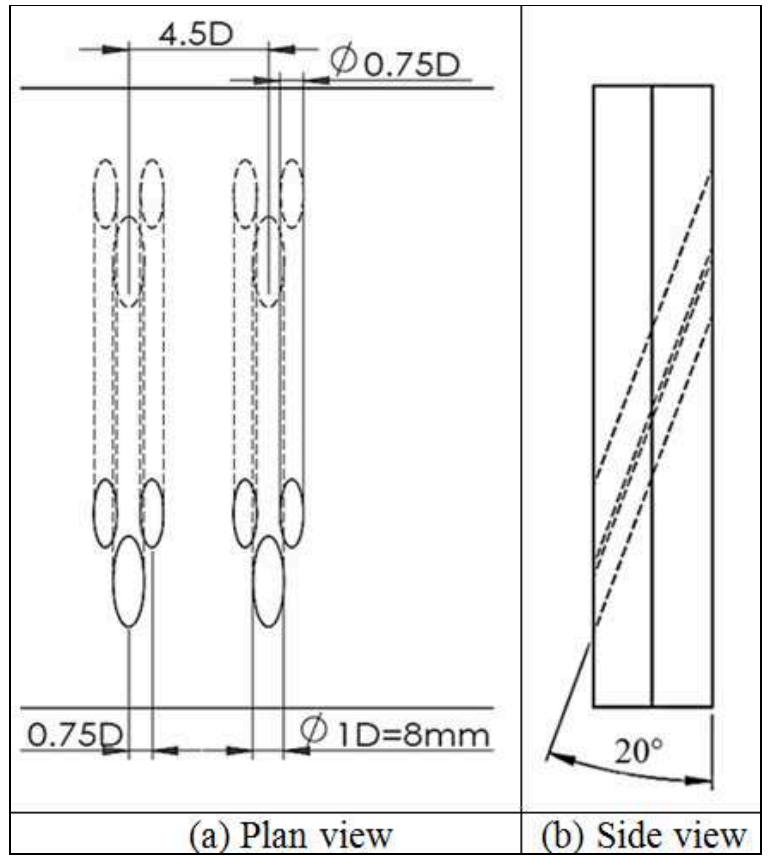

Fig. 2 Geometry of Shallow Hole with Upstream Sister Holes

Film Hole Geometry. The film hole geometry chosen is an array of film holes of $20^{\circ}$ shallow angle with upstream sister holes. The diameter of the main hole, D is $8 \mathrm{~mm}$. Two sister holes with each $0.75 \mathrm{D}$ is placed. They are placed $0.75 \mathrm{D}$ upstream $(x / D)$ and also lateral $(z / D)$ to the main holes. The array consists of 5 sets of sister holes with a pitch of $4.5 \mathrm{D}$. The data is collected from all 5 sets of holes and is compared with Ely et al. [4]. The drawing of the film hole is shown in Figure 2.

Data Analysis. Equation 1 is used to determine the blowing ratio.

$$
M=\frac{\rho_{s} V_{s}}{\rho_{m} V_{m n}}
$$

where $M$ is blowing ratio, $\rho_{s}$ is density of secondary air, $V_{s}$ is velocity of secondary air, $\rho_{m}$ is density of mainstream air and $V_{m}$ is velocity of mainstream air. 
Equation 2 is used to determine the film cooling effectiveness.

$$
\eta=\frac{T_{w}-T_{m}}{T_{s}-T_{m n}}
$$

where $\eta$ is film cooling effectiveness, $T_{w}$ is wall temperature, $T_{m}$ is mainstream temperature, $T_{s}$ is secondary flow temperature.

\section{Results \& Discussion}

The experimental conditions have been planned before the experiment is carried out. However, the actual conditions have deviated a little from the planned conditions. The planned and actual experimental conditions are shown in Table 1.

Table 1 Experimental Conditions

\begin{tabular}{|c|c|c|c|c|}
\hline \multirow{3}{*}{ Planned } & $T_{m}\left({ }^{\circ} \mathrm{C}\right)$ & 30 & 30 & 30 \\
\cline { 2 - 5 } & $T_{S}\left({ }^{\circ} \mathrm{C}\right)$ & 50 & 50 & 50 \\
\cline { 2 - 5 } & $M$ & 1.0 & 1.5 & 2.0 \\
\hline \multirow{3}{*}{ Actual } & $T_{m}\left({ }^{\circ} \mathrm{C}\right)$ & 29 & 29 & 29.5 \\
\cline { 2 - 5 } & $T_{S}\left({ }^{\circ} \mathrm{C}\right)$ & 50.5 & 49 & 49.4 \\
\cline { 2 - 5 } & $M$ & 1.025 & 1.499 & 1.948 \\
\hline
\end{tabular}

Figure 3 shows the laterally averaged effectiveness along the spanwise while Figure 4 show the centre line effectivess along the spanwise. From the figures, $M=1.5$ has the highest effectivess, followed by $M=1.0$ while $M=2.0$ has the lowest effectiveness. These show that $M=1.5$ is the optimum blowing ratio for this configuration of film holes.

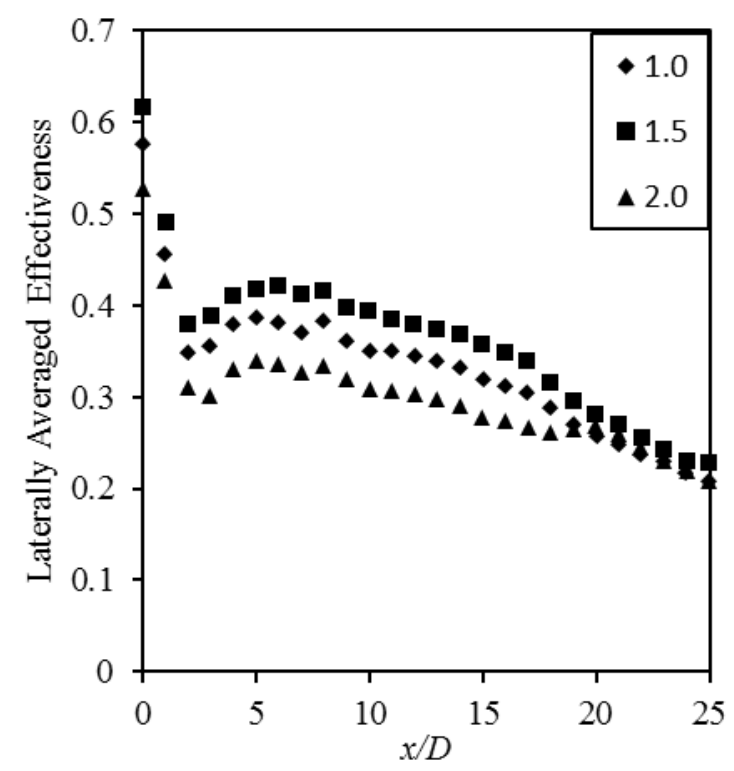

Fig. 3 Laterally Averaged Effectiveness

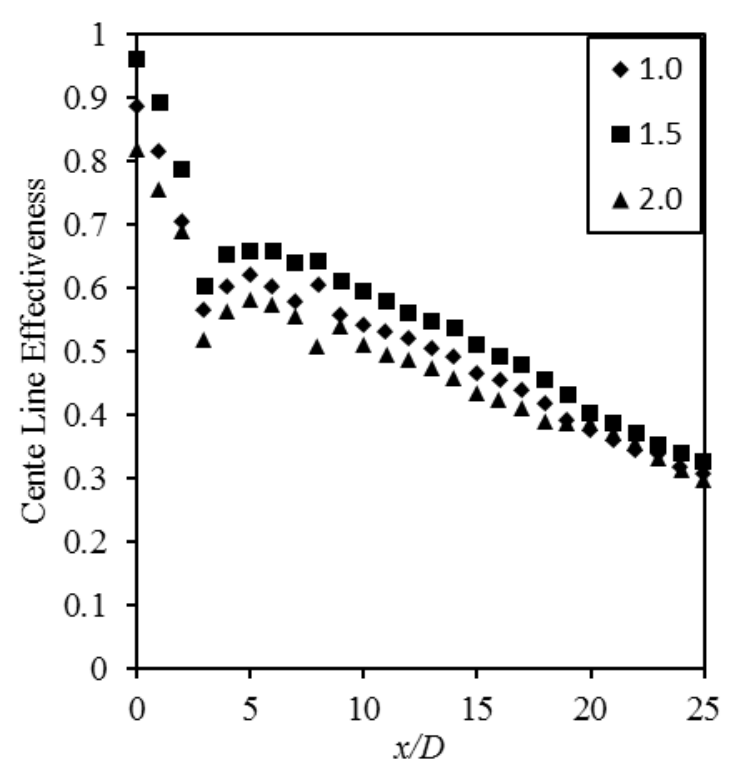

Fig. 4 Centre Line Effectiveness

In the current study, the most important objective is to improve the film cooling effectiveness of shallow angled holes with sister holes proposed by Ely et al. [4]. In order to find improvement of the result, this study proposed a $20^{\circ}$ shallow angle to replace the $35^{\circ}$ shallow angle proposed by Ely et al [4]. Figure 5 and Figure 6 show a significant improvement for both laterally averaged effectiveness and centre line effectiveness for every blowing ratio. With a smaller shallow angle, the lifting effect of secondary flow is reduced. 


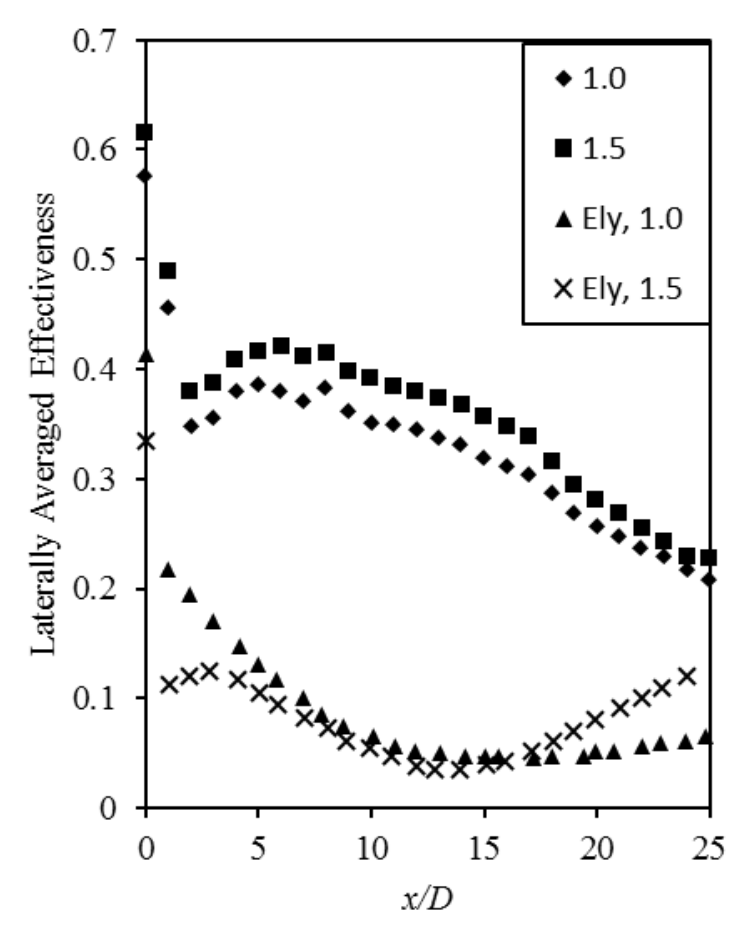

Fig. 5 Comparison of Laterally Averaged Effectiveness

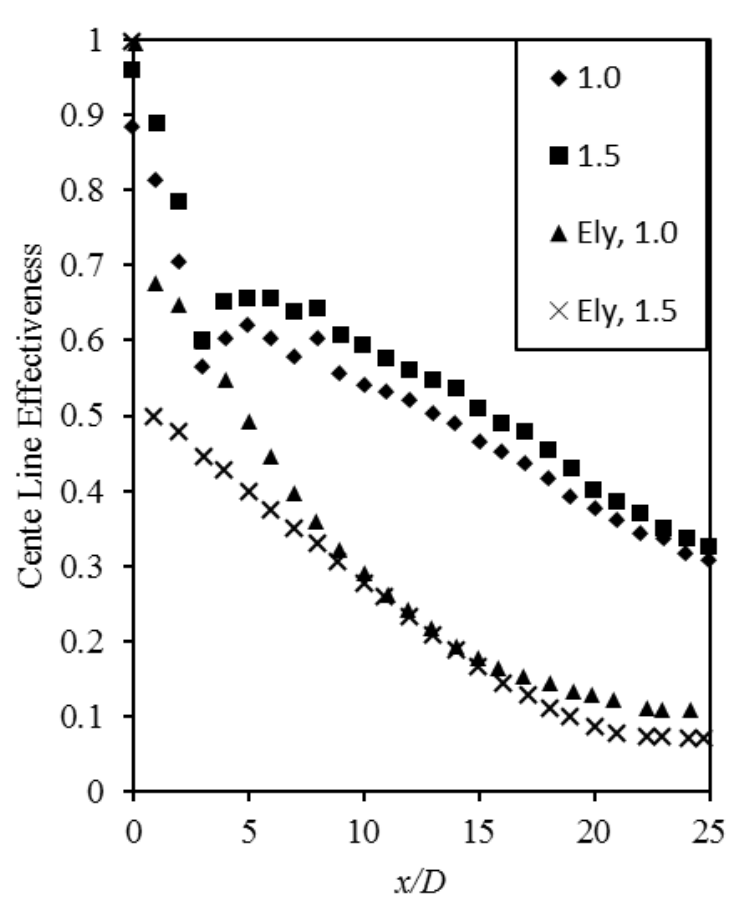

Fig. 6 Comparison of Centre Line Effectiveness

There are few factors which have possibly caused error in the study. First of all, the specification of the Venturi meter is not provided. To overcome this problem, the Venturi meter is calibrated by using a Pitot tube. Furthermore, the reading on the Infrared Camera is not exactly the same with the reading from thermocouple. Therefore, the calibration work has been done before taking thermal images.

\section{Conclusion}

The result showed a significant improvement compared to the previous study due to the use of smaller shallow angle, which is $20^{\circ}$. It is better than a shallow angle of $35^{\circ}$ along the stream wise distance. The optimum blowing ratio is 1.5 for this study. A smaller shallow angle means a smaller injection angle which helps to reduce the jet lifting effect of secondary air flow. The sister holes from upstream help to push the secondary air flow from primary hole onto the wall which increases the film cooling effectiveness.

\section{References}

[1] Han, J., Dutta, S., \& Ekkad, S. (2013). Gas turbine heat transfer and cooling technology. 2nd Edition. Boca Raton: CRC Press, 2012

[2] Goldstein, R. J. (1971). Film cooling. Advances in heat transfer, 7(1), 321-379.

[3] Abdullah, K., Funazaki, K., Onodera, H., \& Ideta, T. (2012). Experimental investigations on aero-thermal interaction of film cooling airs ejected from multiple holes: shallow hole angle. In Proceeding of ASME Turbo Expo.

[4] Ely, M. J., \& Jubran, B. A. (2012). Film cooling from short holes with sister hole influence. In Proceeding of ASME Turbo Expo.

[5] Cengel, Y. A., \& Boles, M. A.. Thermodynamics: an engineering approach. Volume 5. New York: McGraw-Hill. 2011 
[6] Dhungel, A. (2007). Film cooling from a row of holes supplemented with anti vortex holes. Louisiana State University and Agricultural and Mechanical College: Master's Thesis.

[7] Ely, M. J., \& Jubran, B. A. (2009). A numerical study on active film cooling flow control through the use of sister holes. Louisiana State University and Agricultural and Mechanical College: Master's Thesis.

[8] Funazaki, K. I., Kawabata, H., Takahashi, D., \& Okita, Y. (2012). Experimental and numerical studies on leading edge film cooling performance: effects of hole exit shape and freestream turbulence. In Proceeding of ASME Turbo Expo.

[9] Huang, S., \& Liu, Y. (2012). High rotation number effect on heat transfer in a leading edge cooling channel with three channel orientation. In Proceeding of ASME Turbo Expo.

[10]Kröss, B., \& Pfitzner, M. (2012). Numerical and experimental investigation on the film cooling effectiveness and temperature fields behind a novel trench configuration at high blowing ratio. In Proceeding of ASME Turbo Expo.

[11]Liu, C. L., Zhu, H. R., Zhang, Z. W., \& Xu, D. C. (2012). Experimental investigation on the leading edge film cooling of cylindrical and laid-back holes with different hole pitches. International Journal of Heat and Mass Transfer.

[12]Liu, K. C. (2009). Blowing ratio effects on film cooling effectiveness. Texas A\&M University: Master's thesis.

[13] Sakai, E., Takahashi, T., \& Agata, Y. (2012). Experimental study on effects of internal ribs and rear bumps on film cooling effectiveness. In Proceeding of ASME Turbo Expo.

[14] Saravanamuttoo, H. I. H., Rogers, G. F. C., Cohen, C., \& Straznicky, P. V. (2009). Gas turbine theory. 6th ed. Edinburgh Gate: Pearson Education Ltd.

[15]Walsh, P. P., \& Fletcher, P. (2004). Gas turbine performance. 2nd ed. Fairfield: Blackwell Science and ASME. 